\title{
Atypical Femur Fracture Nonunion in a Patient with Osteogenesis Imperfecta
}

\author{
Gaines $\mathrm{DR}^{1}$, Triplet $\mathrm{JJ}^{*}$, Johnson $\mathrm{DB}^{1}$ and Taylor $\mathrm{BC}^{2}$
}

${ }^{1}$ OhioHealth Doctors Hospital, Orthopedic Residency Program, Columbus, OH, United States

${ }^{2}$ OhioHealth Orthopedic Trauma and Reconstruction Surgeons, Grant Medical Center, Columbus, OH, United States

*Corresponding author: Triplet JJ, DO, OhioHealth Doctors Hospital, Orthopedic Residency Program, 100 West Broad Street, USA, Tel: 614-544-2087, E-mail: Jacob.Triplet@ohiohealth.com

Citation: Gaines DR, Triplet JJ, Johnson DB, Taylor BC (2018) Atypical Femur Fracture Nonunion in a Patient with Osteogenesis Imperfecta. J Case Rep Stud 6(4): 404. doi: 10.15744/2348-9820.6.404

Received Date: April 07, 2018 Accepted Date: August 21, 2018 Published Date: August 23, 2018

\begin{abstract}
Case: Here, we present a 43-year-old male with osteogenesis imperfecta (OI) with previously placed intramedullary growing rods that sustained an atypical femur fracture (AFF) following long-term bisphosphonate use. He additionally experienced a non-traumatic ipsilateral femoral neck fracture that was successfully managed with operative stabilization. Despite previous reports following successful conservative management for AFF in patients with OI, nonunion developed requiring osteotomy and surgical stabilization.

Discussion: AFF in patients with OI should be closely monitored for nonunion. Pediatric literature of conservative management may not be applicable to adult patients. Osteotomy and surgical stabilization may be required for fracture union.

Conclusion: Osteotomy and internal fixation utilizing a Dynamic Condylar Screw can be an effective way to achieve union in a subtrochanteric atypical femur fracture in a patient with OI.
\end{abstract}

Keywords: Osteogenesis Imperfecta; Atypical Femur Fracture; Subtrochanteric Femur Fracture; Growing Rods; DCS

List of abbreviations: AFF: Atypical Femur Fracture; OI: Osteogenesis Imperfecta; DCS: Dynamic Condylar Screw; IM: Intramedullary

\section{Introduction}

Osteogenesis Imperfecta (OI) is an inherited defect in type 1 collagen that affects both bone and connective tissues, resulting in an increased incidence of fractures in children. Adolescents with OI are commonly treated with bisphosphonates to increase bone mineral density, reduce the incidence of fracture, and reduce bone pain [1-3]. Treatment of femoral neck fractures in patients with OI treated with cannulated screw fixation around a preexisting intramedullary (IM) rod poses a unique challenge in management, with few reports publishing successful outcomes [4-6] Nonetheless, there are increasing concerns regarding longterm effects of bisphosphonate treatment in adults for osteoporosis, and cases of atypical subtrochanteric femur fractures with long-term bisphosphonate use have been well described in the literature [7-9]. Nicolaou et al., described the pattern of complete subtrochanteric femur fractures that occur with bisphosphonate use in patients with OI, including $38 \%$ occurring in rodded femora [10]. Moreover, AFF have been implicated in patients with OI [11-14]. Incomplete AFF of the subtrochanteric region in patients with OI have been successfully managed non-operatively with administration of teraperatide $[13,15,16]$. However, while there are several reports of AFF occurring in patients with OI treated with bisphosphonates, few cases have reported on the treatment for nonunion in these situations. Successful use of an intramedullary (IM) nail with compression plating has been reported in one case for nonunion [17] and another case described successful treatment with exchange nailing along with medical modalities [11]. However, with previously placed growing rods in this patient; this treatment modality is not applicable. Here, we present a unique case of a 48 year-old male with OI that was successfully managed with operative stabilization of a femoral neck fracture followed by fixation of an ipsilateral AFF nonunion using a dynamic condylar screw (DCS). Appropriate IRB exemption was sought and obtained for the use of information for publication and educational purposes.

\section{Case Report}

A 43-year-old male with a known history of OI was transferred to our facility with a left femoral neck fracture. Previously, he had a 6-week history of left hip pain and was believed to have a stress fracture of his hip. Due to previously placed intramedullary femoral growing rods and incomplete fracture location, conservative management consisting of non-weight bearing (NWB) to his 
left lower extremity for 6 weeks was elected for treatment. After 6 weeks, he sustained a muscle spasm while lying in bed, resulting in immediate pain in his left hip. Radiographs demonstrated a displaced femoral neck fracture as well as a proximal femur stress fracture in the presence of IM growing rods (Figure 1). He was subsequently transferred to our institution for further management. Home medications upon arrival include alendronate $35 \mathrm{mg}$ once weekly.

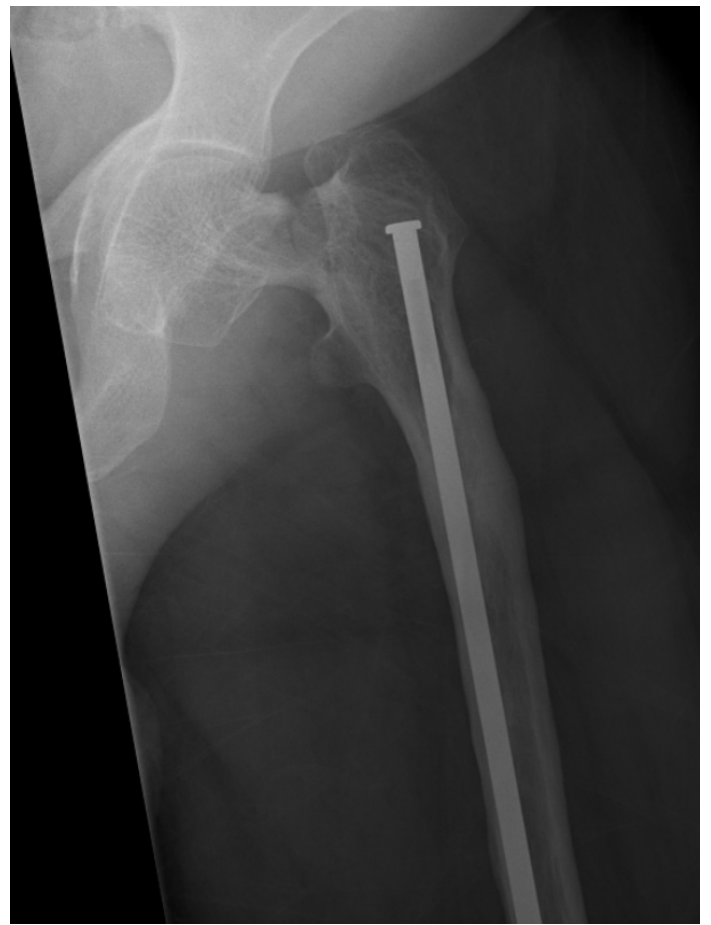

Figure 1: AP radiograph Left hip taken at initial presentation to our institution with femoral neck fracture and incomplete AFF in the presence of previously placed growing femoral rods

The patient was taken to the operating room the following morning for stabilization using three 6.5 mm cannulated screws oriented around the existing IM growing rod. Due to concerns about his healing potential and concurrent stress fracture, the patient was made NWB postoperatively. After discussion with the patient's endocrinologist teriparatide (Forteo) was added to his medication regimen to enhance the fracture healing potential. Postoperative office visits were without issue, and evidence of the stress fracture healing was observed on follow up radiographs (Figure 2a). He was gradually progressed to full weight bearing.

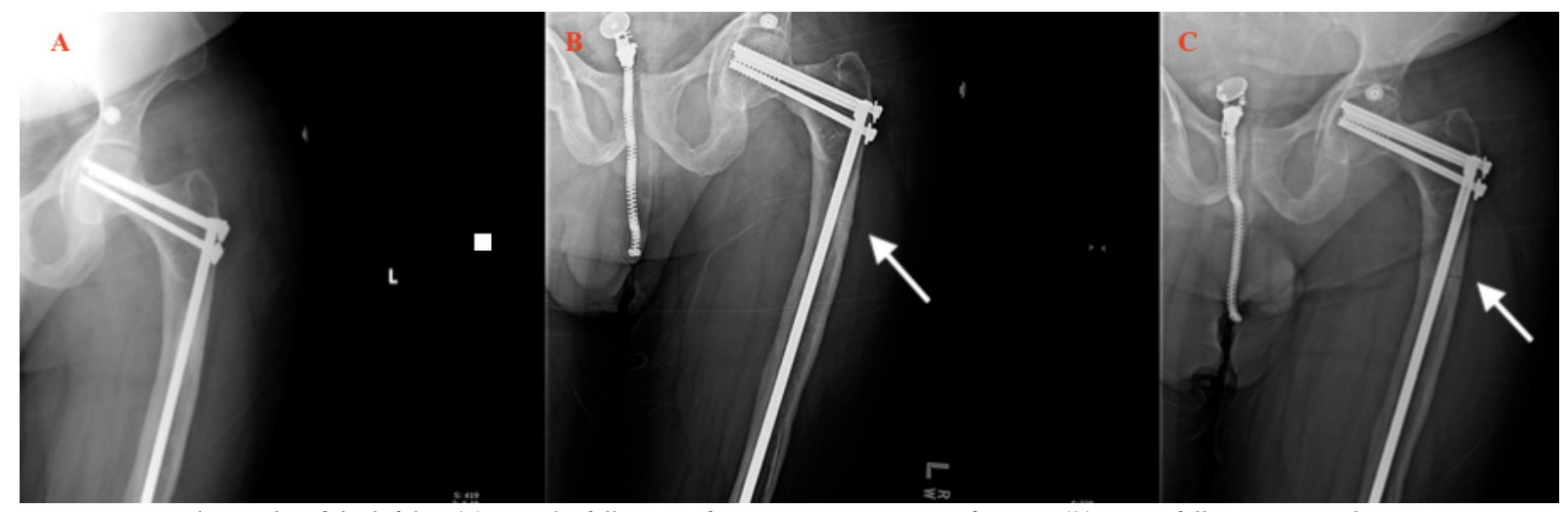

Figure 2: AP radiographs of the left hip (a) 2 weeks follow up after percutaneous screw fixation; (b) 2 year follow up again demonstrating AFF (arrow demonstrating lateral cortical fracture line); (c) 3.5 year follow up, patient with significant pain on ambulation with no healing at the subtrochanteric fracture (arrow demonstrating lateral cortical fracture line)

At three-year follow up, the patient was ambulating without assistive devices. He did complain of thigh soreness that worsened with activity and movement. Radiographs demonstrated a well-healed femoral neck and continued evidence of a stress fracture, improved from prior films (Figure 2b). At 3.5 years after the initial surgery, the patient presented to the office with both thigh and knee pain. He reported the thigh pain to be worse with any attempted ambulation. Radiographs demonstrated nonunion of the stress fracture (Figure 2C). Operative intervention was undertaken, consisting of osteotomy of the left subtrochanteric femur fracture to remove sclerotic bone at the fracture site, internal fixation, removal of hardware, and bone graft placement (Figure $3 \mathrm{a}, \mathrm{b}$ and c). 


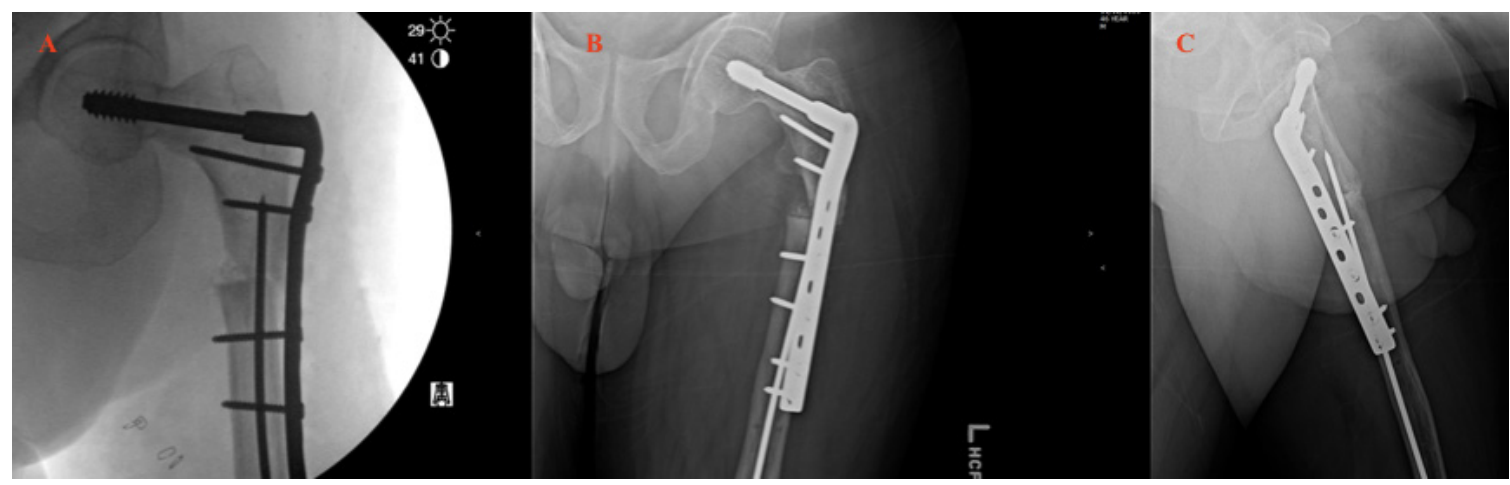

Figure 3: (a) intra-op radiographs osteotomy of fracture site, removal of deep hardware in femoral neck, and removal of proximal aspect of growing rod; (b-c) AP and lateral radiographs at first follow up

At one-year postoperatively, he continued to have minor residual left hip pain and low back pain. He was able to return to his activity of choice, bowling, but with significant start-up pain. At last follow up, he was ambulating unassisted and balance is without issue. Radiographs demonstrate healing at the osteotomy site with DCS in an unchanged position (Figure 4a and b).

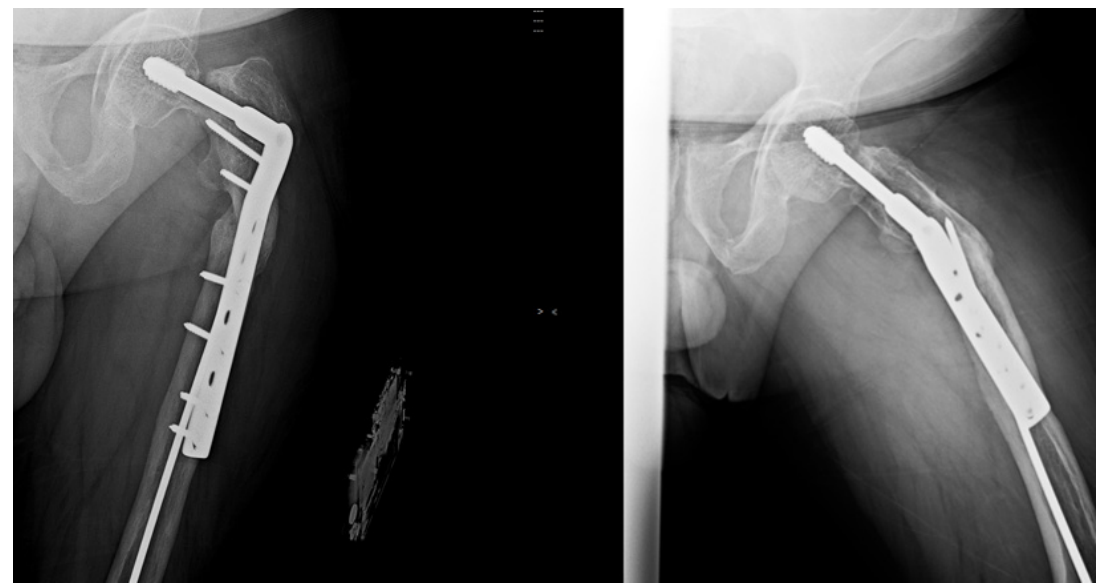

Figure 4: (a-b) AP and lateral radiographs of the left hip at most recent follow up, 5 years after initial presentation at our institution

\section{Discussion}

Atypical femur fractures in patients with OI pose a unique challenge in management for the orthopedic surgeon. Use of a multidisciplinary approach with endocrinology is recommended $[15,16]$. The femoral neck fracture was managed with operative stabilization using cannulated screws around the preexisting IM rod. The difficulty in treating femoral neck fractures around preexisting IM rod in patients with OI has been reported previously in few reports $[4,5,18]$. The ipsilateral incomplete AFF was initially managed conservatively due to fracture location around the IM implant. After failure of the AFF to heal at 3.5 years with conservative management, operative intervention with osteotomy of the fracture site and DCS plate and screw fixation was utilized to achieve union. In this study we discuss the potential for AFF nonunion following long-term bisphosphonate use in a patient with OI after treatment with teraperatide. We encourage continued follow up for OI patients and encourage operative stabilization of symptomatic nonunions.

The difficulty in treating femoral neck fractures in patients with OI receiving bisphosphonates and in the presence of preexisting IM rods has been documented previously in few cases of pediatric patients [4-6]. In these pediatric cases, fixation with cannulated screws successfully achieved fracture union [4-6]. One case report available discusses treatment of a femoral neck fracture in an adult with OI and preexisting IM rod using computer navigation assistance for placement of two cannulated screws [18]. In our case, using intra-operative c-arm with image intensifier, three cannulated screws were able to be passed around the IM rod and achieve good purchase in the femoral head. Follow up in the post-operative months and at 3.5 years demonstrated healing of the femoral neck fracture in the presence of a non-healing AFF in the ipsilateral femur.

The pattern of femoral fractures in patients with OI treated with bisphosphonates was reported by Nicolaou et al., [10] highlighting the tendency for these patients to fracture in the subtrochanteric region, with characteristic bone changes in this region as well. Hegazy et al. [19] reported on 6 cases of children with OI that developed unusual femoral stress fractures. All patients had preexisting IM rods and had been treated for greater than 5 years with bisphosphonates. They identified similar fracture characteristics: subtrochanteric region, transverse or mostly transverse incomplete fractures, IM rod in place, fracture not at a high stress region caused by the IM rod, fracture not located in an area of previous osteotomy, and all had been treated with 
pamidronate for at least 5 years. In their report, all 6 of patients healed with discontinuation of bisphosphonates, conservative management with partial weight bearing, and optimization of vitamin D and calcium. Our case has many similarities to those presented by Hegazy. The fracture is located in the subtrochanteric region, incomplete and transverse, and occurred around a previously placed IM rod. Conversely, we report on an AFF in an adult, 30 years after placement of the IM rods. From the previous reports, the decision was made to manage the AFF conservatively. However, conservative management was unsuccessful, requiring operative stabilization after 3.5 years.

Teriparatide has been described as a treatment modality for AFF related to long-term bisphosphonate use [20] as well as in AFF in patients with OI [12]. In the presence of acute femoral neck fracture with screw stabilization, the fracture healing likely benefited from teraperatide utilization. Here, although follow up radiographs initially did appear to show improvement in the stress fracture following its use, it is still evident and symptomatic in radiographs at 3.5 years. One previous report suggested usage of teriparatide for 2 years followed by 6 months of Denosumab to successfully treat an AFF in a patient with OI [16].

Surgical intervention in femoral neck and AFF have been documented independently in patients with OI. Operative stabilization for AFF in patients with OI has been reported with use of IM nailing [11]. However, to our knowledge, operative stabilization with a DCS following AFF nonunion surrounding a previously placed IM rod has not been reported. Unlike previous reports of AFF in patients with OI with bilateral IM growing rods being successfully managed conservatively [19], we demonstrate that nonunion may ensue and longer-term follow up is merited in patients with OI.

\section{Conclusion}

While no definitive recommendations can be made following a case example, we encourage initial treatment of medical management with multidisciplinary involvement for AFF in patients with OI. A trial of teriparatide has been used successfully in several cases of AFF in patients with OI; this should be considered in patients with history of long standing bisphosphonate use. If conservative measures fail, surgical intervention with osteotomy of the fracture site and use of a DCS plate along with medical management can be a viable option to achieve fracture union.

\section{References}

1. Biggin A, Munns CF (2017) Long-Term Bisphosphonate Therapy in Osteogenesis Imperfecta. Curr Osteoporos Rep 15: 412-8.

2. Tournis S, Dede AD (2017) Osteogenesis imperfecta - A clinical update. Metabolism 80: 27-37.

3. Seikaly MG, Kopanati S, Salhab N, Waber P, Patterson D, et al. (2005) Impact of alendronate on quality of life in children with osteogenesis imperfecta. J Pediatr Orthop 25: 786-91.

4. Tsang KS, Adedapo A (2011) Cannulated screw fixation of fracture neck of femur in children with osteogenesis imperfecta. J Pediatr Orthop B 20: 287-90.

5. Papanna MC, Tafazal S, Bell MJ, Giles SN, Fernandes JA (2017) Femoral neck fractures in osteogenesis imperfecta treated with bisphosphonates. J Child Orthop 11: 191-4.

6. Chow W, Negandhi R, Kuong E, To M (2013) Management pitfalls of fractured neck of femur in osteogenesis imperfecta. J Child Orthop 7: $195-203$.

7. Black DM, Kelly MP, Genant HK, Palermo L, Eastell R, et al. (2010) Bisphosphonates and fractures of the subtrochanteric or diaphyseal femur. N Engl J Med 362: 1761-71.

8. Shane E, Burr D, Abrahamsen B, Adler RA, Brown TD, et al. (2014) Atypical subtrochanteric and diaphyseal femoral fractures: second report of a task force of the American Society for Bone and Mineral Research. J Bone Miner Res 29: 1-23.

9. Miller PD, McCarthy EF (2015) Bisphosphonate-associated atypical sub-trochanteric femur fractures: paired bone biopsy quantitative histomorphometry before and after teriparatide administration. Semin Arthritis Rheum 44: 477-82.

10. Nicolaou N, Agrawal Y, Padman M, Fernandes JA, Bell MJ (2012) Changing pattern of femoral fractures in osteogenesis imperfecta with prolonged use of bisphosphonates. J Child Orthop 6: 21-7.

11. Etxebarria-Foronda I, Carpintero P (2015) An atypical fracture in male patient with osteogenesis imperfecta. Clin Cases Miner Bone Metab 12: 278-81.

12. Holm J, Eiken P, Hyldstrup L, Jensen JE (2014) Atypical femoral fracture in an osteogenesis imperfecta patient successfully treated with teriparatide. Endocr Pract 20: e187-90.

13. Meier RP, Ing Lorenzini K, Uebelhart B, Stern R, Peter RE, et al. (2012) Atypical femoral fracture following bisphosphonate treatment in a woman with osteogenesis imperfecta--a case report. Acta Orthop 83: 548-50.

14. Trejo P, Fassier F, Glorieux FH, Rauch F (2017) Diaphyseal Femur Fractures in Osteogenesis Imperfecta: Characteristics and Relationship With Bisphosphonate Treatment. J Bone Miner Res 32: 1034-39.

15. Watts NB, Aggers D, McCarthy EF, Savage T, Martinez S, et al. (2017) Responses to Treatment With Teriparatide in Patients With Atypical Femur Fractures Previously Treated With Bisphosphonates. J Bone Miner Res 32: 1027-33.

16. Tan JY, Seow CJ (2017) Management of atypical femoral fracture in a patient with osteogenesis imperfecta. BMJ Case Rep 2017: pii: bcr-2017-221835.

17. Grady MK, Watson JT, Cannada LK (2012) Treatment of femoral fracture nonunion after long-term bisphosphonate use. Orthopedics 35: e991-5.

18. Foo CC, Chong KW, Wong MK (2008) A case of screw fixation of femur neck fracture with the use of computer navigation in a woman with osteogenesis imperfecta. J Trauma 65: 1168-70.

19. Hegazy A, Kenawey M, Sochett E, Tile L, Cheung AM, et al. (2016) Unusual Femur Stress Fractures in Children With Osteogenesis Imperfecta and Intramedullary Rods on Long-term Intravenous Pamidronate Therapy. J Pediatr Orthop 36: 757-61.

20. Huang HT, Kang L, Huang PJ, Fu YC, Lin SY, et al. (2012) Successful teriparatide treatment of atypical fracture after long-term use of alendronate without surgical procedure in a postmenopausal woman: a case report. Menopause 19: 1360-3. 


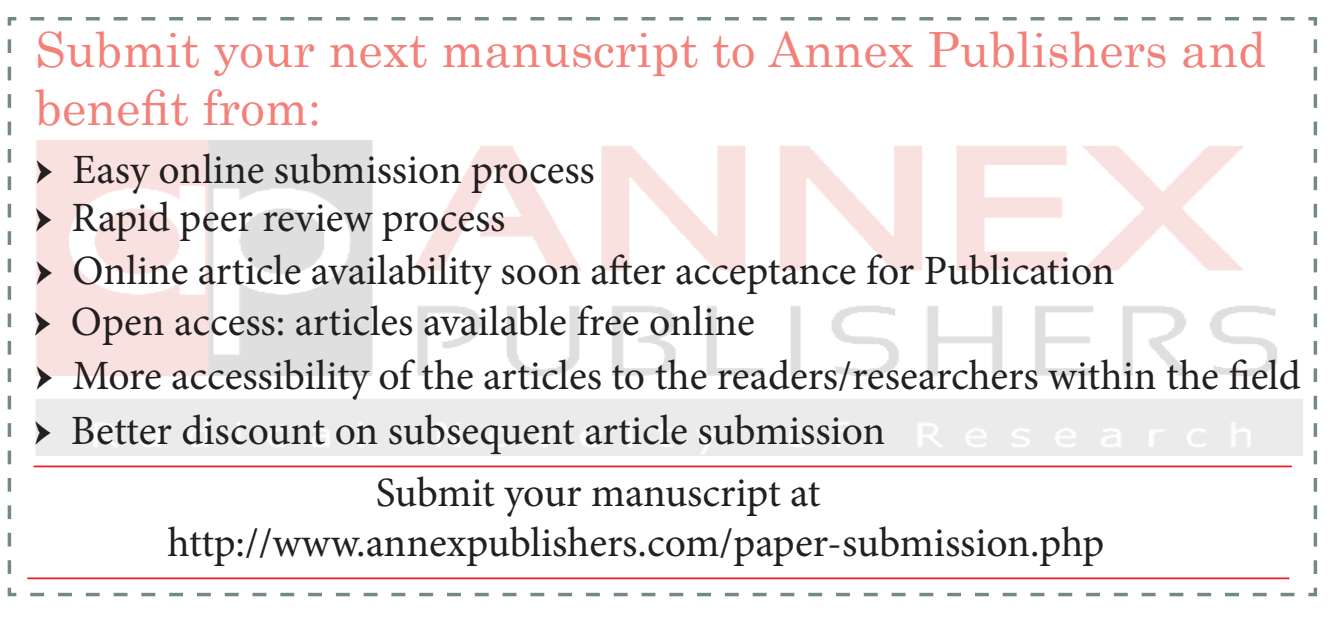

